\title{
Measuring morphology and density of internally mixed black carbon with SP2 and VTDMA: new insight to absorption enhancement of black carbon in the atmosphere
}

\author{
Y. X. Zhang et al. \\ Correspondence to: Q. Zhang (qiangzhang@tsinghua.edu.cn) and H. Su (h.su@mpic.de)
}

The copyright of individual parts of the supplement might differ from the CC-BY 3.0 licence. 
In our study, two types of ambient BC particles is divided based on the delay time distribution, shown in Figure S1. The observed two peaks of delay time distribution is due to the difference in behavior of Ex-BC and In-BC particles during SP2 measurement. The first peak with lower delay time represents the Ex-BC particles. Corresponding, the second peak is considered as the proxy of In-BC particles. Therefore, the particles with delay time more than $1.6 \mu$ s is chose to investigate their core morphology and density.

The LEO fit used for In-BC particles is indirectly checked by that used for ambient non-BC particles (Figure S2). It is widely known that the initial scattering properties of In-BC particles are changed with evaporation of In-BC coatings in SP2 measurement. However, no changes in scattering properties of non-BC particles occurs during SP2 detection due to rarely absorbing components in non-BC particles. Therefore, the scattering properties of non-BC particles derived from LEO fit should be similar with that from scattering signal of SP2 measurement. In our study, the validity of LEO fit used to determine the initial scattering properties of ambient In-BC particles is checked by that used for ambient non-BC particles.

The scattering amplitude obtained from a LEO fit is compared with a full Gaussian fit to the scattering signal detected by the SP2 for ambient non-BC particles, shown in Figure S2. The regression line has a slope of 1.0673, indicating that the scattering properties of non-BC particles from LEO fit was similar with that from SP2 measurement. The good agreement demonstrated that the LEO fit method is valid for ambient particles with an uncertainty of $\sim 6 \%$.

The $\mathrm{RI}_{\text {nonBC }}$ is determined by combining SP2 and VTDMA measurements in our study. In previous $\mathrm{SP} 2$ studies, the $\mathrm{RI}_{\text {nonBC }}$ is usually given an assumption value of around 1.50. The $R I_{\text {nonBC }}$ shown in Figure $\mathrm{S} 3$ is calculated using the scattering properties from SP2 measurement and particle sizes from DMA1, respectively. The $R I_{\text {nonBC }}$ distribution ranges from 1.2 to 1.8 for single charged non-BC particles at 200$350 \mathrm{~nm}$. We selects the peak value of 1.42 as the $R I_{\text {nonBC }}$ for Mie theory calculations. 


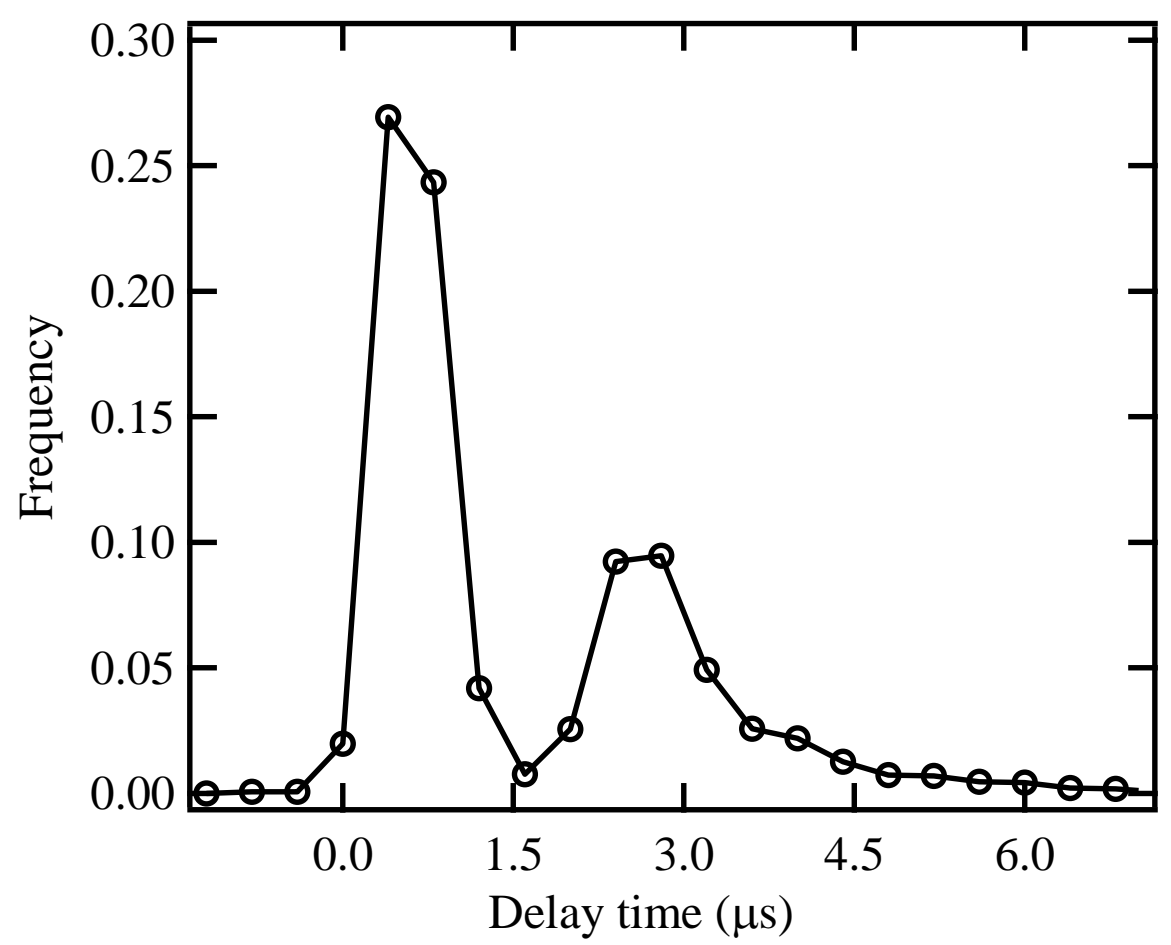

2

Fig. S1. The distribution of delay time in our SP2 measurement. 


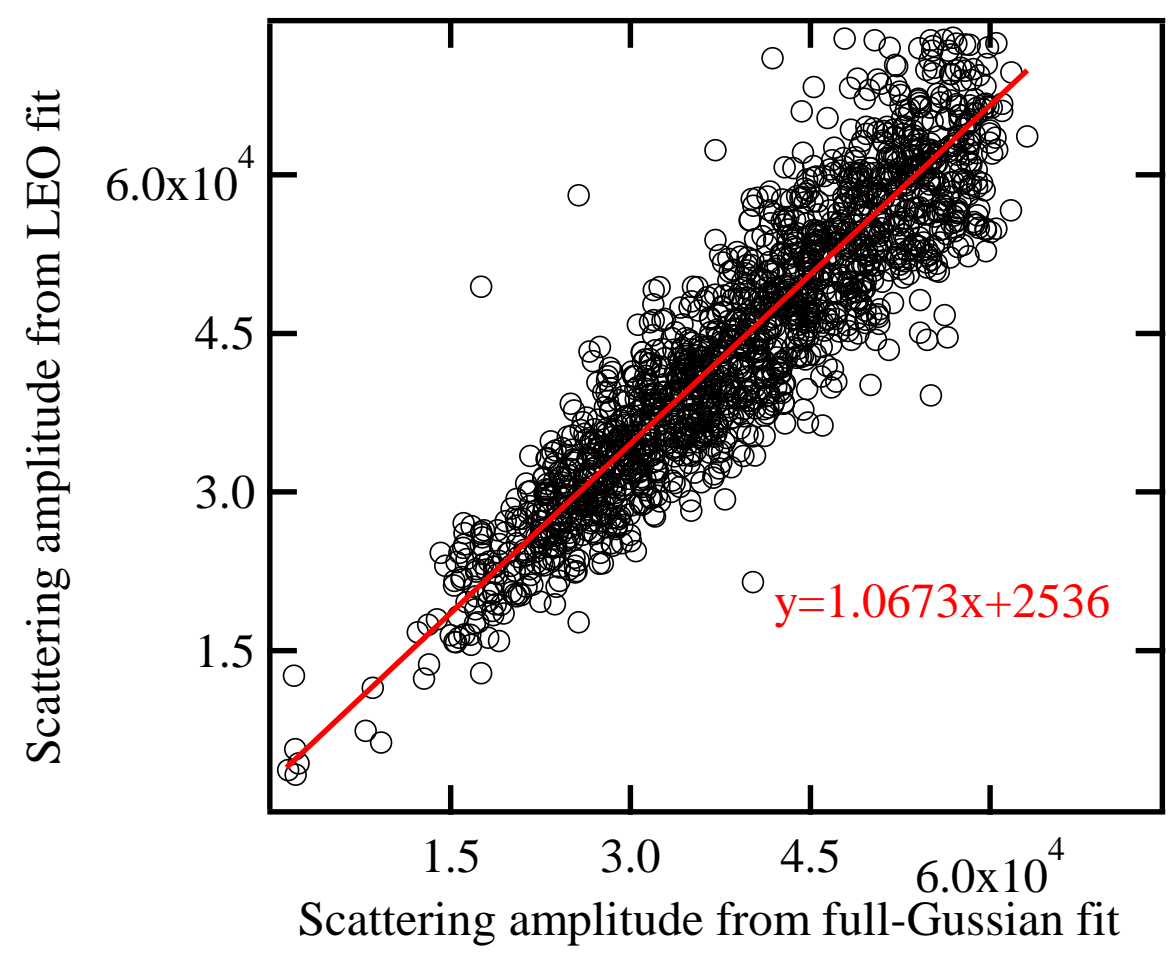

4 Fig. S2. Comparison of scattering amplitude obtained from full Gaussian fit and LEO fit methods 5 for ambient non-BC particles. 


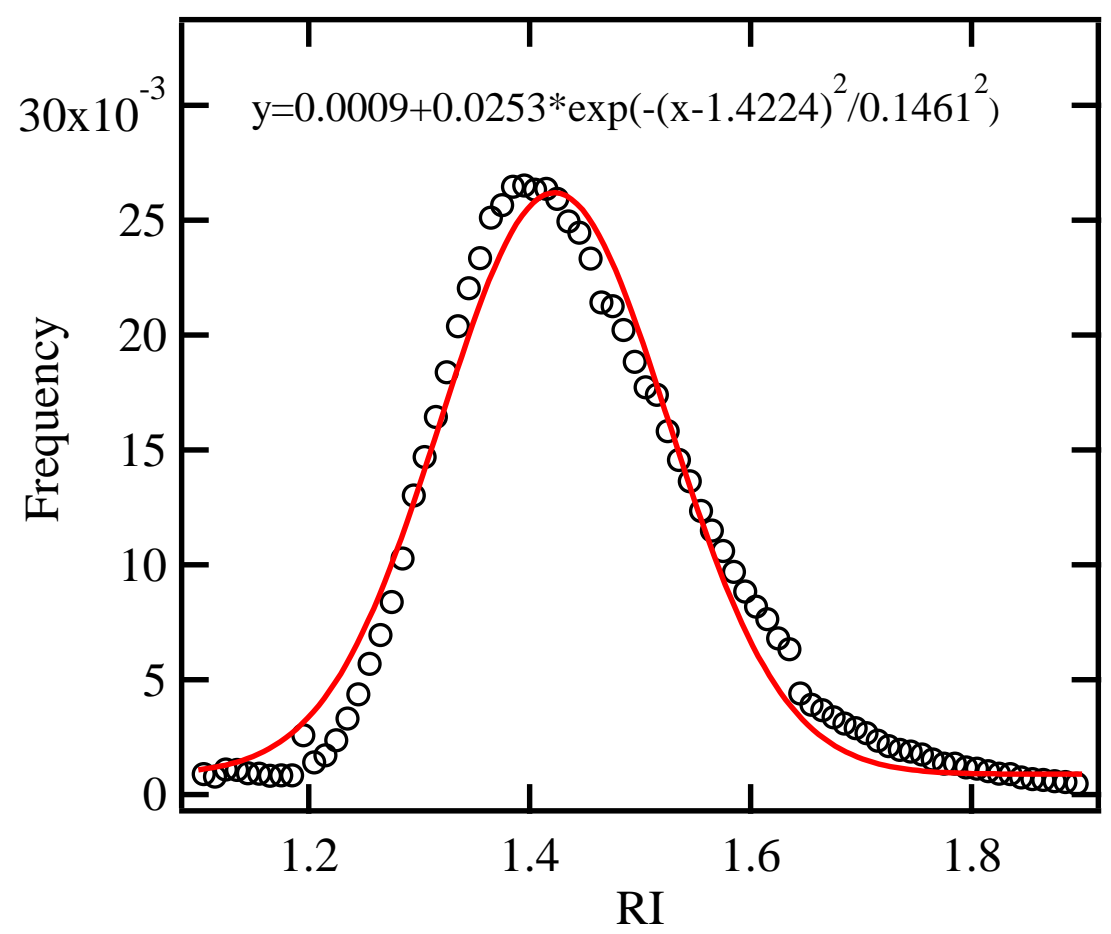

Fig. S3. The RI distribution for non-BC particles. 